\title{
The effects of hand strength on pointing performance
}

\author{
Pradipta Biswas and Peter Robinson \\ University of Cambridge Computer Laboratory \\ $15 \mathrm{JJ}$ Thomson Avenue \\ Cambridge CB3 0FD, UK \\ E-mail: \{pb400,pr\}@cl.cam.ac.uk
}

\begin{abstract}
We have investigated how physical capabilities of users with a wide range of abilities are reflected in their interactions with digital devices. In particular, we have investigated how hand strength affects pointing performance of people with and without motor-impairment for different input devices. Our study indicates that people having higher hand strength also have greater control in hand movement and can perform pointing faster. This result also holds true independently of motor-skills. However we have also found that an appropriate choice of an assistive technology (like a single switch scanning system) can make the interaction speed independent of the physical strength of users.
\end{abstract}

Keywords: Human Computer Interaction, Hand-strength Evaluation, Scanning System, Rapid Aiming Movement.

\section{Introduction}

Pointing tasks form a significant part of human-computer interaction in graphical user interfaces. Fitts' law [3] and its variations [6] are widely used to model pointing as a sequence of rapid aiming movements, especially for able-bodied users. Fitts' Law predicts the movement time as a function of the width and distance to the target. This law is found to be very robust and works in many different situations (even in space and under water). However the application of Fitts' Law for people with motorimpairment is less clear. We have investigated how the interaction of people with motor impairment varies from their able-bodied counterparts. We have investigated how physical strength affects pointing performance of people with and without motorimpairment for different input devices. We measure the physical strength of users by evaluating their hand strength in terms of flexibility and maximum exerted force. It has already been found that the active range of motion (ROM) of the wrist is significantly correlated with movement time in a Fitts' Law task for children with spasticity [9]. Hand evaluation devices are cheap, easy to operate and have good testretest reliability [7]. So these are reliable and useful tools for measuring physical 
strength making these results useful in practice. Our study consisted of the following three experiments:

1. The first experiment involved pointing tasks using a mouse and was undertaken by both motor-impaired and able-bodied participants.

2. The second experiment involved pointing tasks using single switch scanning techniques and was undertaken by both motor-impaired and able-bodied participants.

3. The third experiment involved a 2-dimensional Fitts' Law pointing tasks using a mouse, and was undertaken only by able-bodied participants.

The remainder of this paper presents the experiments in more detail. We hope our study will help in explaining motor action and developing better motor-behaviour models.

\section{Experiment 1: Pointing tasks}

\section{Procedure}

Our study consisted of pointing tasks. A sample screenshot of the task is shown in Figure 1. We followed the description of the multiple tapping tasks in ISO 9241 part 9.In this task the pointer initially located at the middle of the screen. The participants had to move it towards a target (one of the red dots, appearing a light grey in monochrome), and click on it. This process was repeated for all the targets. There were eight targets on the screen and each participant performed the test twice (except participant P2, who retired after completing the first test). The distances to the targets ranged from 200 to 600 pixels while target widths were randomly selected as an integer between 16 and 48 pixels.

\section{Material}

We used a standard optical Mouse and an Acer Aspire 1640 Laptop with a 15.5" monitor having $1280 \times 800$ pixel resolution. We also used the same seating arrangement (same table height and distance from table) for all participants.

We measured the following six variables for hand strength evaluation. Each was measured three times and we took the average. We evaluated only the dominant hand (the hand participants used to operate the mouse). Photographs of the measurement technique can be found at http://www.cl.cam.ac.uk/ pb400/CWUAAT10/ HandStrengthMeasurement.pdf and reference [5].

Grip Strength measures how much force a person can generate by gripping by hand. We measured it using a mechanical dynamometer.

Tip Pinch Strength measures the maximum force generated by a person squeezing something between the tips of his thumb and index finger. We measured it using a mechanical dynamometer. 
The following ranges of motion are defined with respect to the standard anatomical position [5].

Radial deviation is the motion that rotates the wrist away from the midline of the body. We measured the maximum radial deviation using a goniometer.

Ulnar deviation is the motion that rotates the wrist towards the midline of the body. We measured the maximum ulnar deviation using the goniometer.

Pronation is the rotation of the forearm that moves the palm from an anterior-facing position or, palm facing up to a posterior-facing position, or palm facing down. We measured it using a wrist-inclinometer.

Supination is the opposite of pronation, the rotation of the forearm so that the palm faces anteriorly, or palm facing up. We measured it by the wrist-inclinometer.

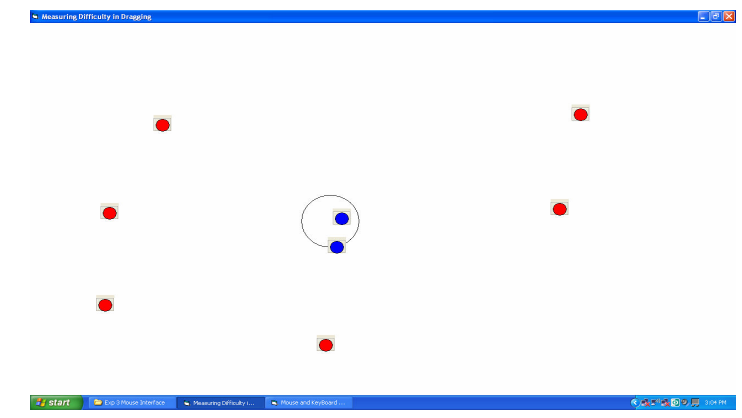

Fig. 1. Screenshot of the experiment for mouse interface

\section{Participants}

We collected data from 10 motor-impaired and 6 able-bodied participants (Table 1). The motor-impaired participants were recruited from a local centre, which works on treatment and rehabilitation of disabled people and they volunteered for the study. To generalize the study, we selected participants with both hypokinetic (e.g. restricted movement, participants P1, P3, P4 etc.) and hyperkinetic (e.g. uncontrolled movement / tremor, participants P5, P6 etc.) movement disorders [4]. All motor-impaired participants used a computer at least once each week. Able-bodied participants were students of our university and expert computer users.

\section{Results}

We found that the movement time significantly correlates $(\rho=0.57, p<0.001)$ with the number of pauses. We defined a pause as an instance while the pointer does not move for more than $100 \mathrm{msec}$. We correlated the average number of pauses per pointing task with the hand strength metrics. Figures 2 to 5 show the graphs of number of pauses with respect to Grip Strength, active ROM of Wrist (Ulnar + Radial Deviation) and active ROM of Forearm (Pronation + Supination) respectively. We found that some users did not have any range of motion in their wrist, though they managed to move the mouse to perform the pointing tasks correctly. We also found 
that the natural logarithm of grip strength (Figure 3 ) significantly correlates with the mean $(\rho=-0.72, p<0.001)$ and standard deviation $(\rho=-0.53, p<0.05)$ of the number of pauses per pointing task. We did not find any correlation between that movement time and the distance, width or Fitts' Law index of difficulty (ID) [3] of the targets for motor-impaired users. This may be due to the presence of physical impairment and the number of pointing tasks (only 16) performed by the participants. We also did not find any significant correlations involving ranges of motion (Figures 4 and 5).

Table 1. List of Participants

\begin{tabular}{|c|c|c|c|}
\hline & Age & Gender & Impairment \\
\hline $\mathrm{C} 1$ & 30 & $\mathrm{M}$ & \\
\hline $\mathrm{C} 2$ & 29 & M & \\
\hline C3 & 28 & $\mathrm{M}$ & \\
\hline $\mathrm{C} 4$ & 25 & M & Able-bodied \\
\hline C5 & 29 & M & \\
\hline C6 & 27 & $\mathrm{~F}$ & \\
\hline P1 & 30 & M & $\begin{array}{l}\text { Cerebral Palsy reduced manual dexterity wheel } \\
\text { chair user. }\end{array}$ \\
\hline $\mathrm{P} 2$ & 43 & M & $\begin{array}{l}\text { Cerebral Palsy reduced manual dexterity also } \\
\text { some tremor in hand wheel chair user. }\end{array}$ \\
\hline P3 & $25-45$ & $\mathrm{~F}$ & $\begin{array}{l}\text { One handed (dominant hand) the other hand is } \\
\text { paralyzed. }\end{array}$ \\
\hline P4 & 30 & M & $\begin{array}{l}\text { Dystonia cannot speak cannot move fingers } \\
\text { wheelchair user. }\end{array}$ \\
\hline P5 & 62 & M & $\begin{array}{l}\text { Left side (non-dominant) paralysed after a stroke } \\
\text { in } 1973 \text { also has tremor }\end{array}$ \\
\hline P6 & 44 & M & $\begin{array}{l}\text { Cerebral attack significant tremor in whole } \\
\text { upper body part fingers always remain folded. }\end{array}$ \\
\hline P7 & 46 & $\mathrm{~F}$ & $\begin{array}{l}\text { Did not mention disease difficulty in gripping } \\
\text { things no tremor. }\end{array}$ \\
\hline P8 & $>45$ & $\mathrm{~F}$ & Spina Bifida/ Hydrocephalus wheelchair user. \\
\hline P9 & 43 & $\mathrm{~F}$ & $\begin{array}{l}\text { Did not mention disease restricted hand } \\
\text { movement no tremor. }\end{array}$ \\
\hline P10 & $>45$ & M & $\begin{array}{l}\text { Cerebral Palsy from birth restricted hand } \\
\text { movement no tremor. }\end{array}$ \\
\hline
\end{tabular}

We divided the whole movement path into three phases [2] and observed how the hand strength affects in the initial, main movement and homing phases. We found that grip strength significantly correlates with the average number of pauses near the source (Figure $6, \rho=-0.61, p<0.01)$ and near the target $(\rho=-0.78, p<0.001)$. We also found that the mean and standard deviation of the velocity of movement were 
significantly correlated with grip strength (Figure 7, $\rho=0.82, \mathrm{p}<0.001$ for mean and $\rho$ $=0.81, \mathrm{p}<0.001$ for standard deviation).

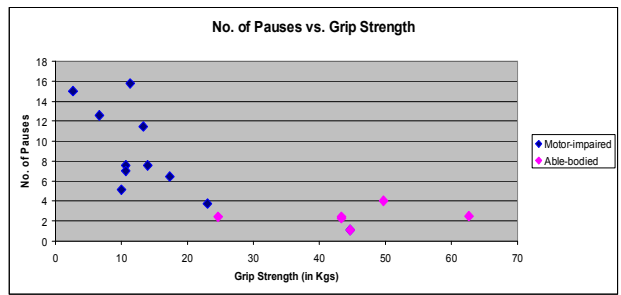

Fig. 2. Average number of Pauses per pointing task vs. Grip Strength

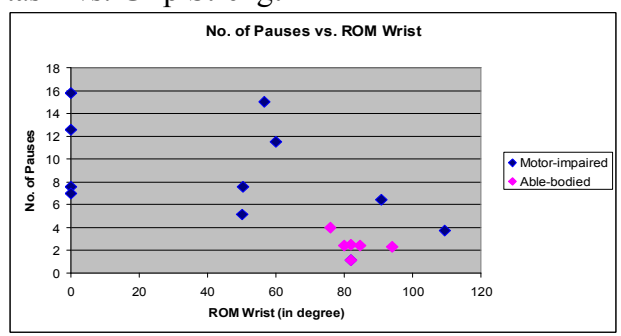

Fig 4. Average number of Pauses per pointing task vs. Active ROM of Wrist

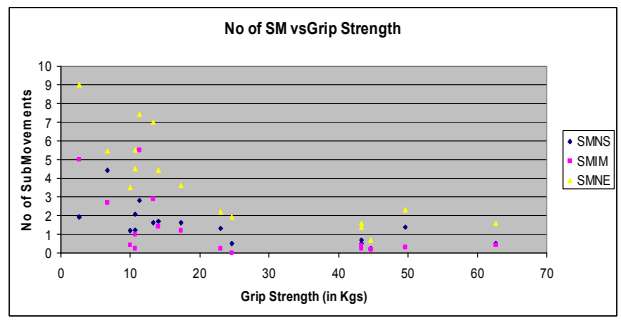

Fig 6. Average number of Pauses per pointing task vs. Grip Strength (SMNS: Sub Movement Near Source, SMIM: SM in Middle SMNE: SM Near End)

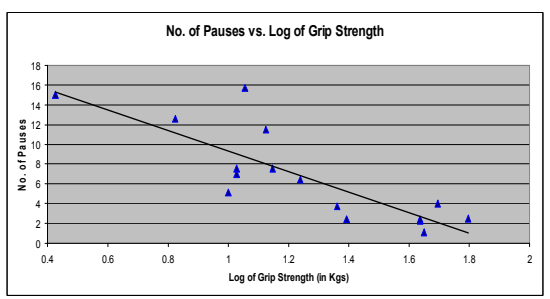

Fig. 3. Average number of Pauses per pointing task vs. Log of Grip Strength

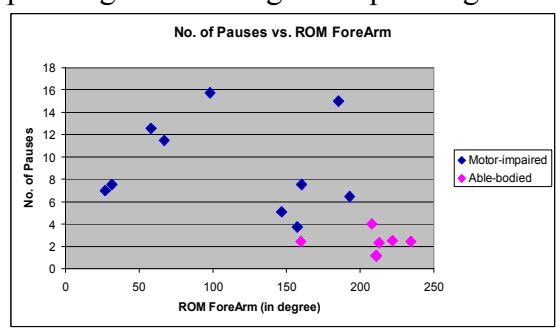

Fig 5. Average number of Pauses per pointing task vs. Active ROM of Forearm

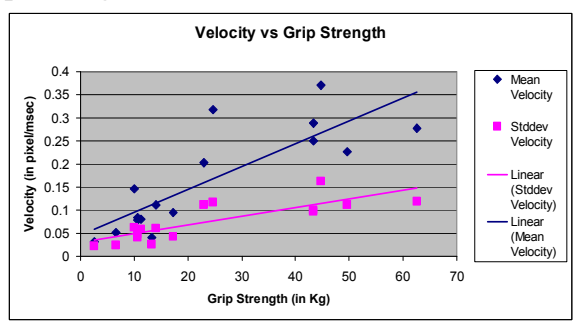

Fig 7. Velocity of Movement vs. Grip Strength

\section{Experiment 2: Scanning study}

Many physically challenged users interact with a computer through one or two switches with the help of a scanning mechanism. Scanning is the technique of successively highlighting items on a computer screen and pressing a switch when the desired item is highlighted. In this study we used the following two scanning systems:

Block Scanning System: A block scanning system iteratively segments the screen into equally sized sub-areas. The user has to select a sub-area that contains the 
intended target. The segmentation process iterates until the sub-area contains a single target.

Cluster Scanning System: A cluster scanning system iteratively divides the screen into several clusters of targets based on their locations. The user has to select the appropriate cluster that contains the intended target. The clustering process iterates until the cluster only contains a single target.

Details of these scanning systems can be found in our previous paper [1].

\section{Procedure}

In this experiment, the participants were instructed to press a set of buttons arranged on a screen (Figure 8) in a particular sequence. All of the buttons were coloured grey except the next target, which was red. After selecting the target its colour changed to grey and another target became red. The same task was repeated for both the scanning systems. We recorded the cursor traces, target height, width, and task completion time. For internal validity of the experiment, the scan delay was kept constant at 2 sec. for all motor-impaired participants and at $1 \mathrm{sec}$. for the control group since the reaction times of motor-impaired users were longer. These values were selected after measuring their reaction times and were greater than the maximum reaction time. All participants were trained adequately with the scanning systems before undertaking the experiment.

\section{Material}

We used a push button switch [10] and an Acer Aspire 1640 Laptop with a 15.5" monitor having $1280 \times 800$ pixel resolution. We used the same seating arrangement for all participants. We measured the same six variables for hand strength evaluation as in Experiment 1.

\section{Participants}

We collected data from 8 motor-impaired (all participants except P3 and P9 in Table 1) and 8 able-bodied participants ( 5 female, 3 male, average age 28.75). The motorimpaired participants were recruited from a local centre and they volunteered for the study. All motor-impaired participants used a computer at least once each week. Able-bodied participants were students of our university and expert computer users. None of the participants had used the scanning systems before.

\section{Results}

We measured the following three variables to investigate the scanning systems. 


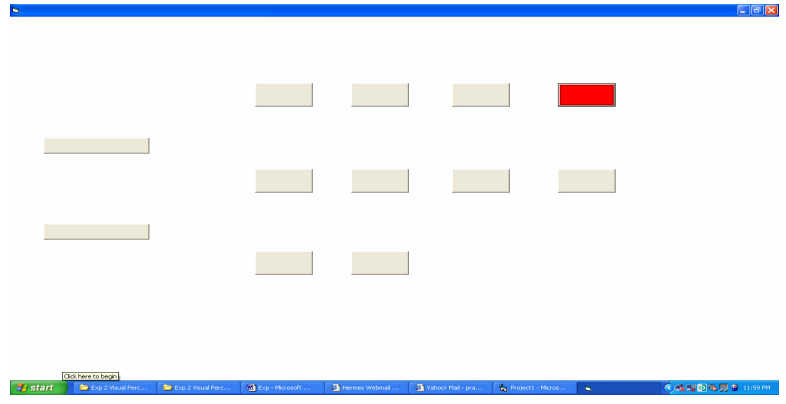

Fig. 8. Screenshot of the experiment

Number of missed clicks: We counted the number of times the participants wrongly pressed the switch.

Idle Count: The scanning systems periodically highlight the buttons. This variable measures the number of cycles when the participants did not provide any input, though they were expected to do so.

Efficiency: The scanning systems require a minimum time to complete any task which depends on the particular scanning system and not on the performance of the user. We calculated the efficiency as the ratio $\frac{\text { OptimalTime }}{\text { ActualTime }}$. An efficiency of $100 \%$ indicates optimal performance, $50 \%$ indicates taking twice the minimal time and $0 \%$ indicates failure to complete the task.

Table 2 shows the correlation coefficients of these variables with the hand evaluation metrics. The only significant effect is a correlation between the number of missed clicks in the cluster scanning system and grip strength, there was a similar, but weaker effect, in the block scanning system. It seems that hand strength does not affect performance of users with the scanning systems. An equal standard deviation t-test did not find any significant difference between the performance of motor-impaired and able-bodied users at the $\mathrm{p}<0.05$ level.

\section{Experiment 3: Fitts' Law study}

We investigated how hand strength affects performance of able-bodied users. Fitts' Law provides a robust and accurate model for rapid aiming movements of ablebodied users. So we conducted a 2-dimensional Fitts' Law task. We used 26 different combinations of target amplitude ( A , ranged from 30 to 700 pixels) and target width ( $\mathrm{W}$, ranged from 16 to 48 pixels). The resulting index of difficulty (ID) ranged from 2 to 5 . Each participant performed 450 pointing tasks. 


\section{Material}

We used a standard optical Mouse and an Acer Aspire 1640 Laptop with 15.5" monitor having $1280 \times 800$ pixel resolution. We also used the same seating arrangement for all participants. We measured the same six variables for hand strength evaluation as in experiment 1.

Table 2. Correlation coefficients for the Scanning Systems

\begin{tabular}{|l|l|r|r|r|r|r|r|}
\hline \multicolumn{2}{|c|}{} & \multicolumn{2}{|l|}{ Cluster Scanning System } & \multicolumn{2}{l|}{ Block Scanning System } \\
\hline \multicolumn{1}{|c|}{} & $\begin{array}{c}\text { Missed } \\
\text { Click }\end{array}$ & $\begin{array}{c}\text { Idle } \\
\text { Count }\end{array}$ & Efficiency & $\begin{array}{c}\text { Missed } \\
\text { Click }\end{array}$ & $\begin{array}{c}\text { Idle } \\
\text { Count }\end{array}$ & Efficiency \\
\hline Correlations & GS & -0.580 & -0.191 & 0.168 & -0.429 & -0.331 & 0.283 \\
\cline { 2 - 9 } & TPS & -0.374 & -0.105 & 0.110 & -0.271 & -0.153 & 0.093 \\
\cline { 2 - 9 } & $\begin{array}{l}\text { ROM } \\
\text { Wrist }\end{array}$ & -0.414 & -0.154 & 0.189 & -0.127 & -0.120 & 0.068 \\
\cline { 2 - 9 } & $\begin{array}{l}\text { ROM } \\
\text { Forearm }\end{array}$ & 0.000 & 0.106 & -0.079 & -0.268 & -0.225 & 0.076 \\
\hline & GS & 0.018 & 0.478 & 0.534 & 0.097 & 0.210 & 0.289 \\
\cline { 2 - 9 } & TPS & 0.153 & 0.699 & 0.686 & 0.310 & 0.572 & 0.731 \\
\cline { 2 - 8 } & $\begin{array}{l}\text { ROM } \\
\text { Wrist }\end{array}$ & 0.111 & 0.569 & 0.484 & 0.639 & 0.659 & 0.803 \\
\cline { 2 - 8 } & $\begin{array}{l}\text { ROM } \\
\text { Forearm }\end{array}$ & 1.000 & 0.695 & 0.770 & 0.315 & 0.401 & 0.778 \\
\hline
\end{tabular}

\section{Participants}

We collected data from 14 able-bodied users ( 9 male, 5 female, and age range 22 to 50 with average age of 29.3). All participants were expert computer users.

\section{Results}

The correlation coefficients between index of difficulty (ID) and movement time ranges from 0.73 to 0.95 with an average value of 0.85 , which conforms to Fitts' Law. We compared the hand evaluation metrics with the Fitts' Law coefficients ( $a$ and $b$ where, $\quad M T=a+b \log _{2}\left(\frac{A}{W}+1\right) \quad$ and Index of Performance $\quad\left(\mathrm{IP}=\frac{I D_{\text {Average }}}{M T_{\text {Averge }}} \quad\right)$. We found that IP is significantly correlated with the grip strength and tip pinch strength ( $\rho=0.57, p<0.05$ for grip strength, $\rho=0.72, p<0.005$ for tip pinch strength, Figures 9 $\& 10$ respectively). The parameter $\mathrm{b}$ significantly correlates with tip pinch strength $(\rho$ $=0.65, \mathrm{p}<0.01$, Figure 11). We did not find any other significant correlation between IP, $\mathrm{a}, \mathrm{b}$ and any other hand evaluation metrics. 


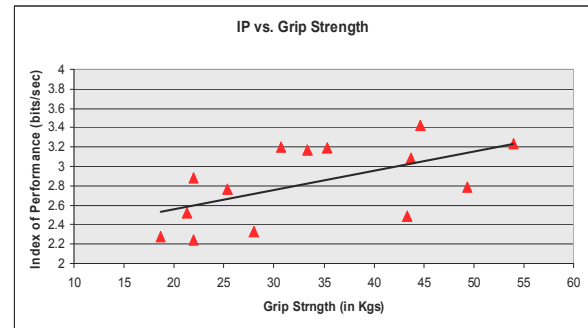

Fig 9. Index of Performance vs. Grip Strength

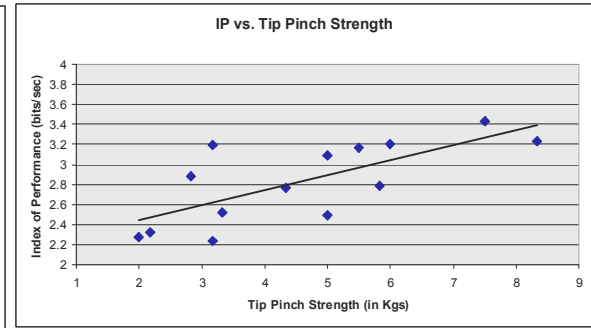

Fig 10. Index of Performance vs. Tip Pinch Strength

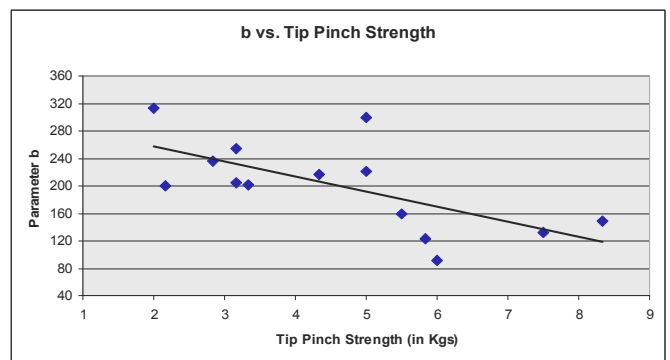

Fig 11. Parameter b vs. Tip Pinch Strength

\section{Discussion}

For able-bodied users, pointing performance is generally analysed in terms of Fitts' Law. Fitts' Law can be applied to rapid aiming movements in many different contexts, but a proper explanation of this law is still unclear. Crossman and Goodeve pioneered an early bit limited mathematical explanation [8]. Meyer et. al. gave a generalized model of rapid aiming movements in which Fitts' Law comes as a special case, however alternative explanations are also available (e.g. the Mass Spring model) [8]. However, Fitts' Law does not account for the users' physical abilities in predicting movement time. This seems reasonable for able-bodied users.

Our analysis indicates that people having higher hand strength also have greater control in hand movement and can perform pointing faster. The positive correlation between the velocity of movement and grip strength also supports this claim. As motor-impairment reduces the strength of a hand, motor-impaired people loose control of hand movement. So the number of pauses near the source and target are significantly affected by grip strength. The logarithmic relation between grip strength and number of pauses indicates that there is a minimum amount of grip strength (about $20 \mathrm{kgs}$ ) required to move the mouse without pausing more than twice. This threshold of $20 \mathrm{~kg}$ can be used to determine the type of input device suitable for a user, along with other factors like preference, expertise etc. Our analysis also showed that flexibility of motion (as measured by ROM of wrist or forearm) is not as important as strength of hand (as measured by grip strength). We found that hand strength affects pointing performance of able-bodied users, too. The positive 
correlation between index of performance and hand strength shows people with greater hand strength perform pointing faster. The correlation between the constant term $b$ and tip pinch strength indicates a difference in movement patterns among people with different hand strengths. As the constant $b$ indicates the effect of index of difficulty $(I D)$ on the movement time, probably the movement pattern of people with higher hand strength mainly consists of an initial ballistic phase and does not have a long homing phase since time to complete the homing phase should depend more on the target characteristics. The opposite holds true for people with less hand strength. As the homing phase requires more control in hand-movement, the negative correlation between $\mathrm{b}$ and hand strength also indicates people having higher hand strength also have greater control in hand movement. We also failed to find any effect of hand strength on pointing performance while participants used the scanning systems. There are two possible explanations:

- The switch used in scanning only requires a gentle push to operate and the hand strength of motor-impaired users are sufficient to operate the switch.

- The scanning software does the navigation itself and the users need not move their hand to move the pointer.

This result with the scanning system also shows that an appropriate choice of an assistive technology can make interaction independent of the physical strength of users.

\section{References}

1. Biswas P. \& Robinson P., A New Screen Scanning System based on Clustering Screen Objects, Journal of Assistive Technologies, Vol. 2 Issue 3 September 2008, pp. 24-31

2. Biswas P. and Robinson P., Automatic Evaluation of Assistive Interfaces, ACM International Conference on Intelligent User Interfaces (IUI) 2008, pp. 247-256

3. Fitts, P.M. The Information Capacity of the Human Motor System In Controlling The Amplitude of Movement. Journal of Experimental Psychology, 47,pp. 381-391, 1954

4. Flowers K. A., Visual 'Closed-Loop' And 'Open-Loop' characteristics Of Voluntary Movement In Patients With Parkinsonism And Intention Tremor, Brain (1976), 99, 269-310

5. Kaplan R. J., Physical medicine and rehabilitation review, 2nd edition, MacGraw Hill, 2006

6. Mackenzie, I. S., Motor Behaviour Models For Human-Computer Interaction. In J. M. Carroll (Ed.) HCI Models, Theories, and Frameworks: Toward A Multidisciplinary Science. pp. 27-54. San Francisco: Morgan Kaufmann, 2003

7. Mathiowetz V., Weber K., Volland G., Kashman N. (1984), Reliability and validity of hand strength evaluation, Journal of Hand Surg, 1984

8. Rosenbaum D. A. , Human Motor Control, Academic Press Inc., USA, 1991

9. Smits-Engelsman B. C. M. et. al., Children with congential spastic hemiplegia obey Fitts' Law in a visually guided tapping task, Journal of Experimental Brain Research (2007), 177, 431-439

10. The Super-Switch (2007), Available at: http://rjcooper.com/super-switch/index.html, Accessed on: 1st July, 2007 